\title{
f-DLPs: Extending Description Logic Programs with Fuzzy Sets and Fuzzy Logic
}

\author{
Tassos Venetis, Giorgos Stoilos, Giorgos Stamou, and Stefanos Kollias, NTUA
}

\begin{abstract}
The Semantic Web can be viewed as largely about "Knowledge meets the Web". Thus its vision includes ontologies and rules. A key requirement for the architecture of the Semantic Web is to be able to layer "rules on top of ontologies" and "ontologies on top of rules". This has as a counterpart the definition of a mapping between Description Logics and Logic Programming, which is known as Description Logic Programs. In this paper we extend the Description Logic Programs with fuzzy sets and fuzzy logic in order to be able to represent the imprecision and vagueness of real-life applications. We provide the common semantics of the mapping, and the conditions that must be met for this semantic equivalence, based on the modeltheoretic semantics.
\end{abstract}

\section{INTRODUCTION}

The Semantic Web [1] is a mesh of information where data is organized in a machine understandable way and where Semantic Web agents are able to (semi)automatically carry out complex tasks assigned by humans in a meaningful (semantic) way. The vision of the Semantic Web includes ontologies as well as rules.

An ontology is a controlled vocabulary that describes objects and relations between them in a formal way and has a grammar for using the vocabulary terms to express something meaningful, within a specified domain of interest. Ontologies are extremely useful for the explicit definition of terms commonly used by different web sources. Ontologies are mostly expressed through OWL (Web ontology Language) [2], [3], which has been recently standardized by W3C. It consists of three increasingly expressive sublanguages, namely OWL Lite, OWL DL and OWL Full. OWL Lite and $O W L D L$ are, basically very expressive description

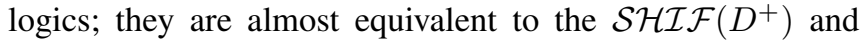
$\mathcal{S H O} \mathcal{I} \mathcal{N}\left(D^{+}\right)$Description Logics [4]. However, despite its expressive power regarding other languages, such as RDF [5], OWL has some expressive limitations. Rules, that are more expressive, allow for the modelling of knowledge by implication rules, a feature missing in OWL. Def-Horn rules, used in this paper, are the fragment of Horn rules that are definite, negated predicates and negation-as-failureliterals are not allowed in the rules, and equality free, Horn rules that do not include the equality predicate.

In order to combine the advantages of ontologies and rules a mapping between Description Logics and Horn rules has been proposed, known as Description Logic Programs [6].

Tassos Venetis, Giorgos Stoilos, Giorgos Stamou and Stefanos Kollias are with the Department of Electrical and Computer Engineering, National Technical University of Athens, Iroon Polytechniou 9 street, Zographou 15780, Greece, avenet@image.ntua.gr, gstoil@image.ntua.gr, gstam@softlab.ntua.gr, stefanos@cs.ntua.gr
This approach concerns the study of the fragment of OWL that is semantically equivalent to def-Horn rules and is different from the approach proposed by [7] which concerns dl-programs, that consist of a description logic knowledge base and a finite set of description logic rules that is the union of Description Logics and Logic Programming.

Description Logic Programs (DLPs) are the intersection of First Order Logic (FOL) and Logic Programming and to be more precise DLPs are the intersection of OWL and def-Horn rules. They are ontological knowledge bases which lie within the intersection of OWL and Logic Programming. They are created by the DLP-fusion: the bidirectional translation of premises and inferences from the DLP fragment of Description Logics to Logic Programming, and vise versa from the DLP fragment of Logic Programming to Description Logics. Syntactically, DLPs are a fragment of OWL, in the sense that every DLP knowledge base is a syntactically valid Description Logics knowledge base. Semantically, they carry the semantics of OWL DL and each DLP knowledge base is equivalent (semantically) to a set of Horn clauses of First Order Logic.

However, DLPs cannot represent imprecise knowledge. Knowledge is organized or contextualized information which can be used to produce new meanings and generate new data. Knowledge is inherently imprecise and vague, like the concepts of a "tall" person, a "nice" car, a "beautiful" woman and others, therefore the use of fuzzy concepts and rules results to more realistic applications. The need of studying imprecise knowledge for the Semantic Web has been stressed out many times in the literature over the last years [8][10]. In this paper we extend DLPs to Fuzzy DLPs in order to represent uncertain, vague or imprecise knowledge. Our approach is different from [11] which introduces fuzzines for dl-programs and [12] that introduces the syntax and semantics of a novel fuzzy version of the nominal construct. The rest of the paper is organized as follows. Section II provides brief introductions to fuzzy OWL and fuzzy def-Horn rules, as well as their model-theoretic semantics. Section III provides the mapping between fuzzy OWL and fuzzy defHorn rules and in Sect. IV we define the languages produced from this mapping. Finally, Sect. V concludes the paper.

\section{PRELIMINARIES}

\section{A. Fuzzy Description Logics and OWL}

In this section, we provide an introduction to a fuzzy extension of OWL DL by adding degrees to OWL facts; we call this extension f-OWL. This extension is based on Fuzzy Sets and Fuzzy Logic [13] and on previous work on 
TABLE I

FUZZY OWL ClASS DESCRIPTIONS AND AXIOMS

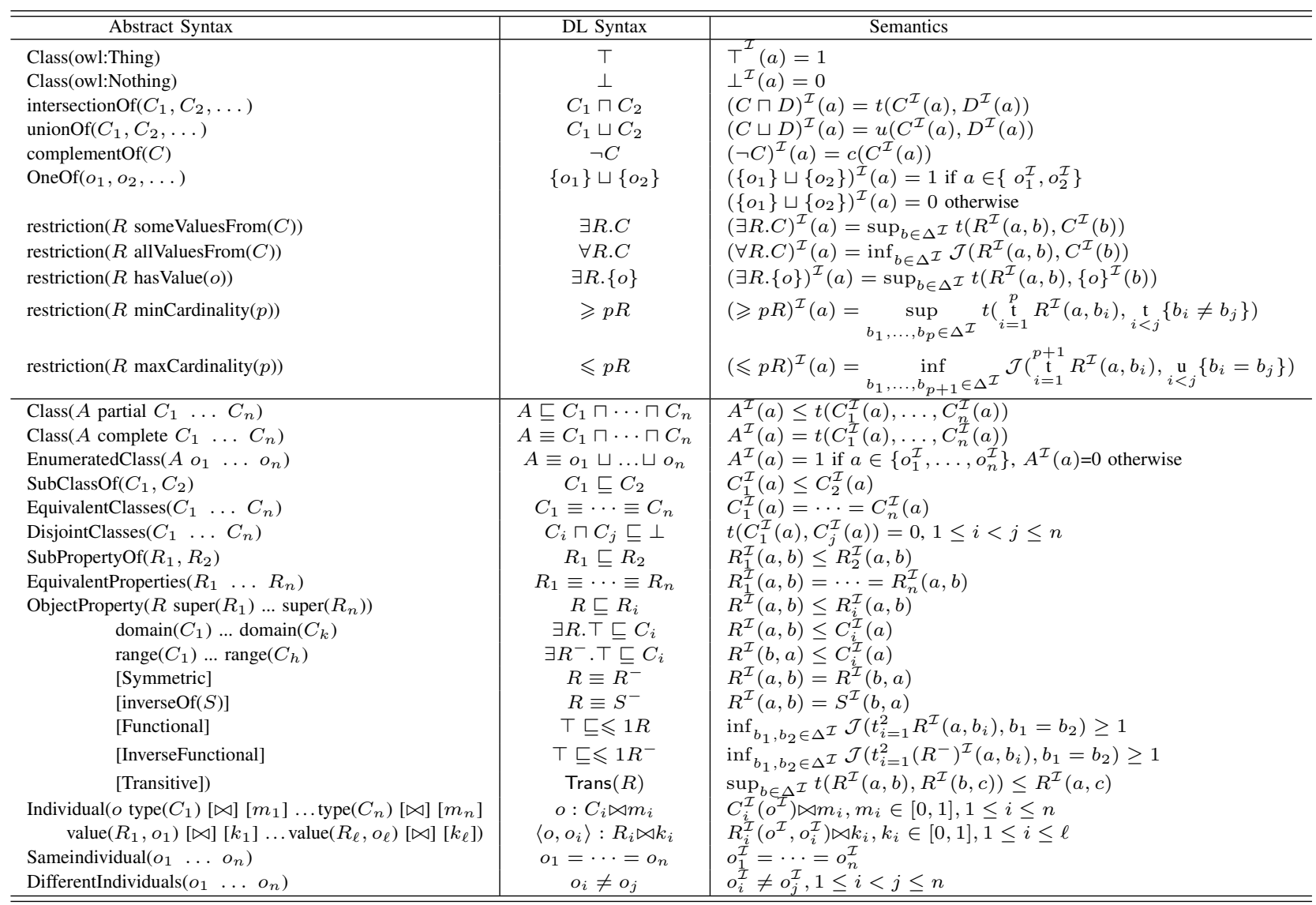

fuzzy Description Logics [14], [15] and fuzzy OWL [16]. However, in this extension there is no use of datatypes, as it is an ongoing research problem.

As usual fuzzy DLs (fuzzy OWL) are defined by an alphabet of distinct concept names (class names) $\mathrm{C}$, role names (property names) $\mathbf{R}$ and individuals $\mathbf{I}$. The set of roles (properties) is defined as $\mathbf{R} \cup\left\{R^{-} \mid R \in \mathbf{R}\right\}$, where $R^{-}$ represents the inverse of $R$. By using concept constructors we can define concept descriptions. More precisely, if $o \in \mathbf{I}$, $A \in \mathbf{C}, R \in$ Roles, $S$ a simple role [2] and $p \in \mathbb{N}$, then $\mathrm{f}$ $\mathcal{S H O I N}$-concepts are defined inductively by the following abstract syntax:

$$
\begin{aligned}
C, D \longrightarrow & \perp|\top| A|C \sqcup D| C \sqcap D|\neg C| \forall R . C \mid \\
& \exists R . C|\geq p S . C| \leq p S . C \mid\{o\}
\end{aligned}
$$

A fuzzy DL Knowledge Base consists of a TBox, RBox and ABox. A TBox is a set of concept subsumptions of the form, $C \sqsubseteq D$ and concept equivalences of the form $C \equiv D$, where $C, D$ are $\mathrm{f}-\mathcal{S H O} \mathcal{H} \mathcal{N}$-concepts, an RBox is a set of transitive role axioms of the form $\operatorname{Trans}(R)$ and role subsumptions of the form $R \sqsubseteq S$, where $R, S$ are $\mathrm{f}-\mathcal{S H O I N}$-roles, while an ABox is a set of concept and role assertions of the form $(a: C) \bowtie n$ and $(\langle a, b\rangle: R) \bowtie n$, or individual equalities and inequalities of the form $a=b$ or $a \neq b$, where $a, b \in \mathbf{I}$, $\bowtie \in\{\geq,>, \leq,<\}$ and $n \in[0,1]$. As stated above, OWL and DLs have a strong correspondence. More precisely, all constructors of OWL can be expressed by DL expressions. In Table I we can see the abstract syntax and the corresponding DL syntax for fuzzy OWL class descriptions and axioms, where $R$ is a property name, $C, C_{1}, \ldots, C_{n}$ are concept descriptions, $o, o_{1}, \ldots, o_{n}$ are individuals, $p \in \mathbb{N}$, and $m_{i}, k_{j} \in[0,1]$.

The semantics of f-OWL are based on fuzzy interpretations. A fuzzy interpretation $\mathcal{I}$ is a pair $\mathcal{I}=\left(\Delta^{\mathcal{I}},{ }^{\mathcal{I}}\right)$, where the domain $\Delta^{\mathcal{I}}$ is, like the crisp case, a non-empty set of objects and ${ }^{\mathcal{I}}$ is a fuzzy interpretation function, which maps

- an individual name o to an object $o^{\mathcal{I}} \in \Delta^{\mathcal{I}}$,

- a concept name $C$ to a membership function $C^{\mathcal{I}}$ : $\Delta^{\mathcal{I}} \rightarrow[0,1],{ }^{1}$ and

- a property name $R$ to a membership function $R^{\mathcal{I}}: \Delta^{\mathcal{I}} \times$ $\Delta^{\mathcal{I}} \rightarrow[0,1]$

The fuzzy interpretation is extended to give semantics to fuzzy concepts and property descriptions presented in Table $\mathrm{I}$, where sup is the supremum, inf is the infimum, $c$ is a fuzzy complement, $t$ is a fuzzy conjunction (t-norm), $u$ is a fuzzy disjunction (t-conorm) and $\mathcal{J}$ is a fuzzy implication.

${ }^{1}$ For instance, given an object $a \in \Delta^{\mathcal{I}}$ and a class name $C, C^{\mathcal{I}}(a)$ gives a degree of confidence (such as 0.8 ) that the object $a$ belongs to the fuzzy concept $C$. 
There are some remarks regarding Table I. Firstly, most of the semantics have been presented elsewhere [14], [16]. In contrast to [14] and [16] we use the revised semantics for cardinality restrictions (concepts of the form $\geqslant p R$ and $\leqslant p R$ ) presented in [15]. Moreover, the semantics of the fuzzy axiom $\exists R . \top \sqsubseteq C_{i}$ result from the semantics of the expressions $\exists R$. $\top$ and $C_{i} . \exists R . \top$ is interpreted as $(\exists R . \top)^{\mathcal{I}}(a)=\sup _{b \in \Delta^{\mathcal{I}}} t\left(R^{\mathcal{I}}(a, b), 1\right)$ which is equivalent to $(\exists R \cdot \top)^{\mathcal{I}}(a)=\sup _{b \in \Delta^{\mathcal{I}}} R^{\mathcal{I}}(a, b)$ and $C_{i}$ as $C_{i}^{\mathcal{I}}(a)$. Thus, the semantics of the fuzzy axiom $\exists R . \top \sqsubseteq C_{i}$ are $C_{i}^{\mathcal{I}}(a) \geq \sup _{b \in \Delta^{\mathcal{I}}} R^{\mathcal{I}}(a, b)$. This is simplified to the expression $C_{i}^{\mathcal{I}}(a) \geq R^{\mathcal{I}}(a, b)$ which is equivalent to $R^{\mathcal{I}}(a, b) \leq$ $C_{i}^{\mathcal{I}}(a)$.

\section{B. Fuzzy def-Horn rules}

In this section we provide a short introduction to a fuzzy extension of def-Horn rules by adding degrees to facts. This extension is called fuzzy def-Horn rules.

Let $\{P, Q, \ldots\}$ be a set of predicates, $\{\leftarrow, \wedge\}$ be a set of operators, $\{\vec{x}, \vec{y}, \ldots\}$ be a set of tuples of variables or constants and $\{\bar{r}, \bar{z}, \ldots\}$ be a set of unary constant truth values. A fuzzy def-Horn rule is defined as the expression

$$
\text { Head } \leftarrow \text { Body }
$$

where the Body consists of positive predicates, that is predicates without negation connected with the intersection $\wedge$ operation, or a constant truth value. The Head consists of a single positive predicate or a constant truth value. So, fuzzy def-Horn rules are of the form $P(\vec{y}) \leftarrow Q_{1}\left(\overrightarrow{x_{1}}\right) \wedge Q_{2}\left(\overrightarrow{x_{2}}\right)$ $\wedge \ldots \wedge Q_{n}\left(\overrightarrow{x_{n}}\right), P(\vec{y}) \leftarrow \bar{r}$ or $\bar{r} \leftarrow Q(\vec{x})$. An atom $t$ is either a variable, a constant or an expression of the form $P\left(t_{1}, \ldots, t_{n}\right)$, where $t_{1}, \ldots, t_{n}$ are terms. A collection of fuzzy def-Horn rules is a fuzzy def-Horn program.

The semantics of fuzzy def-Horn rules are based on fuzzy interpretations. A fuzzy interpretation $\mathcal{I}$ is a pair $\mathcal{I}=$ $\left(\Delta^{\mathcal{I}},{ }^{\mathcal{I}}\right)$, where the domain $\Delta^{\mathcal{I}}$ is, like the crisp case, a non-empty set of objects called the Herbrand Universe of the fuzzy def-Horn program and ${ }^{\mathcal{I}}$ is a fuzzy interpretation function, which maps

- a variable or constant $x$ to an object $x^{\mathcal{I}} \in \Delta^{\mathcal{I}}$,

- a constant truth value $\bar{r}$ to a real number $r \in[0,1]$,

- a predicate $P$ to a membership function $P^{\mathcal{I}}: \Delta^{\mathcal{I} n} \rightarrow$ $[0,1]$.

So, the rule $P(\vec{y}) \leftarrow Q_{1}\left(\overrightarrow{x_{1}}\right) \wedge Q_{2}\left(\overrightarrow{x_{2}}\right) \wedge \ldots \wedge Q_{n}\left(\overrightarrow{x_{n}}\right)$ based on the model-theoretic semantics, is interpreted with the inequality

$$
P^{\mathcal{I}}\left(\vec{y}^{\mathcal{I}}\right) \geq t\left(Q_{1}^{\mathcal{I}}\left(\vec{x}_{1}^{\mathcal{I}}\right), Q_{2}^{\mathcal{I}}\left({\overrightarrow{x_{2}}}^{\mathcal{I}}\right), \ldots, Q_{n}^{\mathcal{I}}\left({\overrightarrow{x_{n}}}^{\mathcal{I}}\right)\right),
$$

the rule $\bar{r} \leftarrow Q_{1}\left(\overrightarrow{x_{1}}\right) \wedge Q_{2}\left(\overrightarrow{x_{2}}\right) \wedge \ldots \wedge Q_{n}\left(\overrightarrow{x_{n}}\right)$ with the inequality

$$
r \geq t\left(Q_{1}^{\mathcal{I}}\left({\overrightarrow{x_{1}}}^{\mathcal{I}}\right), Q_{2}^{\mathcal{I}}\left({\overrightarrow{x_{2}}}^{\mathcal{I}}\right), \ldots, Q_{n}^{\mathcal{I}}\left({\overrightarrow{x_{n}}}^{\mathcal{I}}\right)\right),
$$

and the rule $P(\vec{y}) \leftarrow \bar{r}$ with the inequality

$$
P^{\mathcal{I}}\left(\vec{y}^{\mathcal{I}}\right) \geq r
$$

where $t$ is a t-norm.

The Herbrand Base of a fuzzy def-Horn program is the set of ground instantiations of all the atoms appearing in the program, and by ground we mean the instantiations obtained by replacing all variables with constants from the Herbrand Universe.

In the following we will only use predicates with a maximum arity of two, as this is the maximum arity of fuzzy OWL predicates, that is the arity of fuzzy OWL constants, concepts and properties.

\section{MAPPING F-OWL TO FUZZY DEF-HORN RULES}

In this section we provide the mapping, semantic equivalence check, between f-OWL and fuzzy def-Horn rules, based on the model-theoretic semantics. According to this mapping in the next section we define fuzzy Description Horn Logic (DHL) ontologies as the set of fuzzy Description Logics axioms that can be mapped to fuzzy def-Horn rules without loss of their semantics. Based on this definition we, moreover, define fuzzy Description Logic Programs as the Logic Programming ruleset that are the logic equivalent to a DHL set.

\section{A. Mapping Axioms}

Axioms in Description Logics concern subsumption relationships and definitions, according to the TBox of the Knowledge Base, as well as assertions, according to the Abox of the Knowledge Base. For the following mapping we use $C, D$ to describe fuzzy concepts (predicates with one variable) and $P, Q$ to describe fuzzy roles (predicates with two variables). Moreover, the fuzzy interpretation of fuzzy Description Logics are according to Table I and the fuzzy interpretation of fuzzy def-Horn rules according to 1-3.

First of all, we check the mapping regarding subsumption relationships. The fuzzy axiom of the form $C \sqsubseteq D$, which declares that concept $C$ is subsumed by concept $D$, is interpreted as $C^{\mathcal{I}}(a) \leq D^{\mathcal{I}}(a)$. Since this interpretation holds for every model we can educe the fuzzy def-Horn rule $D(x) \leftarrow$ $C(x)$. Thus, there is a semantic equivalence between the fuzzy DLs axiom $C \sqsubseteq D$ and the fuzzy def-Horn rule $D(x) \leftarrow C(x)$. The fuzzy axiom of the form $Q \sqsubseteq P$, which declares that role $Q$ is subsumed by $P$, is interpreted as $Q^{\mathcal{I}}(a, b) \leq P^{\mathcal{I}}(a, b)$. Since this interpretation holds for every model we can educe the fuzzy def-Horn rule $P(x, y) \leftarrow$ $Q(x, y)$, which is, thus, semantically equivalent to the fuzzy axiom $Q \sqsubseteq P$. The fuzzy axiom of the form $\exists P . \top \sqsubseteq C$, which declares that the domain of role $P$ is the concept $C$, is interpreted as $\sup _{b \in \Delta^{\mathcal{I}}} t\left(P^{\mathcal{I}}(a, b), 1\right) \leq C^{\mathcal{I}}(a)$. This interpretation can be simplified to $t\left(P^{\mathcal{I}}(a, b), 1\right) \leq C^{\mathcal{I}}(a)$ which is equivalent to $P^{\mathcal{I}}(a, b) \leq C^{\mathcal{I}}(a)$. Since this fuzzy interpretation holds for every model we can educe the fuzzy def-Horn rule $C(x) \leftarrow P(x, y)$. In the same way the fuzzy axiom of the form $\exists P^{-} . \top \sqsubseteq C$, which declares that the range of role $P$ is the concept $C$, is interpreted as $\left.\sup _{b \in \Delta^{\mathcal{I}}} t\left(\left(P^{-1}\right)^{\mathcal{I}}\right)(a, b), 1\right) \leq C^{\mathcal{I}}(a)$ that is equivalent to $\sup _{b \in \Delta^{\mathcal{I}}} t\left(P^{\mathcal{I}}(b, a), 1\right) \leq C^{\mathcal{I}}(a)$. This interpretation can be simplified to $t\left(P^{\mathcal{I}}(b, a), 1\right) \leq C^{\mathcal{I}}(a)$ which is equivalent 
to $P^{\mathcal{I}}(b, a) \leq C^{\mathcal{I}}(a)$. Since this fuzzy interpretation holds for every model we can educe the fuzzy def-Horn rule $C(y) \leftarrow P(x, y)$. The transitivity axiom of the form $P^{+} \sqsubseteq P$ is interpreted as $\sup _{b \in \Delta^{\mathcal{I}}} t\left(P^{\mathcal{I}}(a, b), P^{\mathcal{I}}(b, c)\right) \leq P^{\mathcal{I}}(a, c)$ which is equivalent to $t\left(P^{\mathcal{I}}(a, b), P^{\mathcal{I}}(b, c)\right) \leq P^{\mathcal{I}}(a, c)$. The later results to the fuzzy def-Horn rule $P(x, z) \leftarrow$ $P(x, y) \wedge P(y, z)$. Thus, there exists semantic equivalence between the fuzzy axiom $P^{+} \sqsubseteq P$ and the fuzzy def-Horn rule $P(x, z) \leftarrow P(x, y) \wedge P(y, z)$.

Moreover, we check the mapping regarding fuzzy definitions of concepts and roles. The fuzzy definition of the form $C \equiv D$, which declares that concepts $C$ and $D$ are equivalent, is interpreted as $C^{\mathcal{I}}(a)=D^{\mathcal{I}}(a)$. This is equivalent to $C^{\mathcal{I}}(a) \leq D^{\mathcal{I}}(a)$ and $D^{\mathcal{I}}(a) \leq C^{\mathcal{I}}(a)$, from which we can educe the fuzzy def-Horn ruleset $D(x) \leftarrow$ $C(x)$ and $C(x) \leftarrow D(x)$. Consequently, there exists a mapping between $C \equiv D$ and the fuzzy def-Horn ruleset $D(x) \leftarrow C(x)$ and $C(x) \leftarrow D(x)$. Following the same procedure we show the equivalence of the interpretations in case of fuzzy roles. The fuzzy axiom $P \equiv Q$ is interpreted as $P^{\mathcal{I}}(a, b)=Q^{\mathcal{I}}(a, b)$, which is equivalent to $Q^{\mathcal{I}}(a, b) \leq$ $P^{\mathcal{I}}(a, b)$ and $P^{\mathcal{I}}(a, b) \leq Q^{\mathcal{I}}(a, b)$. These two inequalities result to the fuzzy def-Horn ruleset $P(x, y) \leftarrow Q(x, y)$ and $Q(x, y) \leftarrow P(x, y)$, thus, there exists semantic equivalence between $P \equiv Q$ and the fuzzy def-Horn ruleset $P(x, y) \leftarrow$ $Q(x, y)$ and $Q(x, y) \leftarrow P(x, y)$. The fuzzy axiom of the form $P \equiv Q^{-1}$ that declares that role $P$ is the inverse of role $Q$ is interpreted as $P^{\mathcal{I}}(a, b)=\left(Q^{-1}\right)^{\mathcal{I}}(a, b)=Q^{\mathcal{I}}(b, a)$. This interpretation is equivalent to the set of interpretations $Q^{\mathcal{I}}(b, a) \leq P^{\mathcal{I}}(a, b)$ and $P^{\mathcal{I}}(a, b) \leq Q^{\mathcal{I}}(b, a)$. Since these hold for every model we can educe the fuzzy def-Horn ruleset $P(x, y) \leftarrow Q(y, x)$ and $Q(y, x) \leftarrow P(x, y)$, which is semantically equivalent to the fuzzy axiom $P \equiv Q^{-1}$.

Finally, asserted class-instance (type) and instanceproperty-instance relationships, which correspond to fuzzy DL axioms $o: C \bowtie m, m \in[0,1]$ and $\left(o_{1}, o_{2}\right): P \bowtie k, k \in$ $[0,1]$ respectively, are interpreted as $C^{\mathcal{I}}\left(o^{\mathcal{I}}\right) \bowtie m, m \in[0,1]$ and $P^{\mathcal{I}}\left(o_{1}^{\mathcal{I}}, o_{2}^{\mathcal{I}}\right) \bowtie k, k \in[0,1]$. The corresponding fuzzy defHorn rules educed from these semantics are $C(x) \leftarrow \bar{r}$ and $P(x, y) \leftarrow \bar{r}$ for $\bowtie=\geq$ and $\bar{r} \leftarrow C(x)$ and $\bar{r} \leftarrow P(x, y)$, for $\bowtie=\leq$. For $\bowtie=<$ or $\bowtie=>$ no def-Horn rules can be educed.

\section{B. Mapping Class Constructors}

In the previous section we have showed how fuzzy Description Logic axioms correspond with fuzzy def-Horn rules. However in fuzzy Description Logics classes appearing in these statements need not be atomic, but can be complex, compound expressions build up from atomic classes and properties using a variety of constructors. This is why we continue by studying the mapping between constructors of f-OWL and fuzzy def-Horn rules.

1) Conjunction $(\sqcap)$ : A f-OWL class can be formed by conjoining existing classes, e.g. $C \sqcap D$. This corresponds to the conjunction of unary predicates. Conjunction can be directly expressed in the body of a fuzzy def-Horn rule. A conjunction that occurs in the left hand side of a subclass axiom of the form $C_{1} \sqcap C_{2} \sqsubseteq D$ is interpreted as $t\left(C_{1}^{\mathcal{I}}(a), C_{2}^{\mathcal{I}}(a)\right) \leq D^{\mathcal{I}}(a)$. As this interpretation holds for every model we can educe the rule $D(x) \leftarrow C_{1}(x) \wedge C_{2}(x)$, which is also interpreted as $t\left(C_{1}^{\mathcal{I}}(a), C_{2}^{\mathcal{I}}(a)\right) \leq D^{\mathcal{I}}(a)$.

When the conjunction occurs in the right hand side of a subclass axiom of the form $C \sqsubseteq D_{1} \sqcap D_{2}$ the fuzzy interpretation is $C^{\mathcal{I}}(a) \leq t\left(D_{1}^{\mathcal{I}}(a), D_{2}^{\mathcal{I}}(a)\right)$. As from this interpretation we cannot educe any def-Horn rule we will have to simplify $C^{\mathcal{I}}(a) \leq t\left(D_{1}^{\mathcal{I}}(a), D_{2}^{\mathcal{I}}(a)\right)$, according to the property of fuzzy t-noms $t(a, b) \leq a, b$ to the set of interpretations $C^{\mathcal{I}}(a) \leq D_{1}^{\mathcal{I}}(a)$ and $C^{\mathcal{I}}(a) \leq D_{2}^{\mathcal{I}}(a)$. However, the equivalence of $C^{\mathcal{I}}(a) \leq D_{1}^{\mathcal{I}}(a)$ and $C^{\mathcal{I}}(a) \leq$ $D_{2}^{\mathcal{I}}(a)$ to $C^{\mathcal{I}}(a) \leq t\left(D_{1}^{\mathcal{I}}(a), D_{2}^{\mathcal{I}}(a)\right)$, stands only for the Gödel t-norm (min) as it is the only idempotent t-norm $(t(a, a)=a)$. Thus, using only the Gödel's t-norm, we can educe, from the fuzzy interpretations $C^{\mathcal{I}}(a) \leq D_{1}^{\mathcal{I}}(a)$ and $C^{\mathcal{I}}(a) \leq D_{2}^{\mathcal{I}}(a)$, the fuzzy def-Horn ruleset $D_{1}(x) \leftarrow C(x)$ and $D_{2}(x) \leftarrow C(x)$. Therefore, we can see that there exists a semantic equivalence concerning fuzzy intersection only when the Gödel's t-norm is used.

2) Union ( $\sqcup$ ): A f-OWL class can be formed from a disjunction of existing classes, e.g. $C \sqcup D$. This corresponds to the disjunction of unary predicates. A disjunction that occurs in the left hand side of a subclass axiom of the form $C_{1} \sqcup C_{2} \sqsubseteq D$ is interpreted as $D^{\mathcal{I}}(a) \geq u\left(C_{1}^{\mathcal{I}}(a), C_{2}^{\mathcal{I}}(a)\right)$ which according to the property of fuzzy t-conorms $u(a, b) \geq$ $a, b$ is equivalent to the set of interpretations $D^{\mathcal{I}}(a) \geq$ $C_{1}^{\mathcal{I}}(a)$ and $D^{\mathcal{I}}(a) \geq C_{2}^{\mathcal{I}}(a)$. However, the reciprocal, the equivalence of $D^{\mathcal{I}}(a) \geq C_{1}^{\mathcal{I}}(a)$ and $D^{\mathcal{I}}(a) \geq C_{2}^{\mathcal{I}}(a)$ to $D^{\mathcal{I}}(a) \geq u\left(C_{1}^{\mathcal{I}}(a), C_{2}^{\mathcal{I}}(a)\right)$ stands only for the Gödel's tconorm, as it is the only idempotent t-conorm $(u(a, a)=a)$. Thus, using only the Gödel's t-conorm, we can educe, from the fuzzy interpretations $C_{1}^{\mathcal{I}}(a) \leq D^{\mathcal{I}}(a)$ and $C_{2}^{\mathcal{I}}(a) \leq$ $D^{\mathcal{I}}(a)$, the fuzzy def-Horn ruleset $D(x) \leftarrow C_{1}(x)$ and $D(x) \leftarrow C_{2}(x)$. Therefore, we can see that there exists a semantic equivalence concerning fuzzy union only when the Gödel's t-conorm is used.

When the disjunction occurs in the right hand side of a subclass axiom of the form $D \sqsubseteq C_{1} \sqcup C_{2}$ the fuzzy interpretation is $D^{\mathcal{I}}(a) \leq u\left(C_{1}^{\mathcal{I}}(a), C_{2}^{\mathcal{I}}(a)\right)$. From this interpretation we educe the fuzzy def-Horn rule of the form $C_{1}(x) \vee C_{2}(x) \leftarrow D(x)$, which is not regarded as a fuzzy def-Horn rule. So, in general, the f-OWL axiom $D \sqsubseteq C_{1} \sqcup C_{2}$ cannot be mapped to a fuzzy def-Horn rule.

3) Universal restriction $(\forall)$ : In Description Logics the universal quantifier can only be used in restrictionsexpressions of the form $\forall P . C$. This is equivalent to a First Order Logic clause of the form $\forall y . P(x, y) \rightarrow C(y)$ which means that every $y$ for which $P(x, y)$ is valid is an instance of the concept $C$. A universal quantifier that occurs in the right hand side of a subclass axiom of the form $D \sqsubseteq \forall P . C$ is interpreted as $D^{\mathcal{I}}(a) \leq$ $\inf _{b \in \Delta^{\mathcal{I}}} \mathcal{J}\left(P^{\mathcal{I}}(a, b), C^{\mathcal{I}}(b)\right)$. This interpretation can be simplified to $D^{\mathcal{I}}(a) \leq \mathcal{J}\left(P^{\mathcal{I}}(a, b), C^{\mathcal{I}}(b)\right)$ Using the property of $R$-implications " $t(a, b) \leq d$ iff $\mathcal{J}_{R}(a, d) \geq b$ " the fuzzy interpretation $D^{\mathcal{I}}(a) \leq \mathcal{J}\left(P^{\mathcal{I}}(a, b), C^{\mathcal{I}}(b)\right)$ can be 
TABLE II

Mapping Between FuZzy Description Logic Axioms And FuZZy DeF-Horn Rules

\begin{tabular}{|c|c|c|}
\hline Fuzzy DL Axioms & Fuzzy def-Horn Rules & Common Semantics \\
\hline$o: C \bowtie \bar{r}$ & $C(x) \leftarrow \bar{r}$ & $C^{\mathcal{I}}\left(o^{\mathcal{I}}\right) \geq r, r \in[0,1](\bowtie=\geq)$ \\
\hline & $\bar{r} \leftarrow C(x)$ & $C^{\mathcal{I}}\left(o^{\mathcal{I}}\right) \leq r, r \in[0,1](\bowtie=\leq)$ \\
\hline$\left\langle o_{1}, o_{2}\right\rangle: P \bowtie \bar{r}$ & $P(x, y) \leftarrow \bar{r}$ & $P^{\mathcal{I}}\left(o_{1}^{\mathcal{I}}, o_{2}^{\overline{\mathcal{I}}}\right) \geq r, r \in[0,1](\bowtie=\geq)$ \\
\hline & $\bar{r} \leftarrow P(x, y)$ & $P^{\mathcal{I}}\left(o_{1}^{\mathcal{I}}, o_{2}^{\overline{\mathcal{I}}}\right) \leq r, r \in[0,1](\bowtie=\leq)$ \\
\hline$C \sqsubseteq D$ & $D(x) \leftarrow C(x)$ & $C^{\mathcal{I}}(a) \leq D^{\overline{\mathcal{I}}}(a)$ \\
\hline$Q \sqsubseteq P$ & $P(x, y) \leftarrow Q(x, y)$ & $Q^{\mathcal{I}}(a, b) \leq P^{\mathcal{I}}(a, b)$ \\
\hline$\exists P . \top \sqsubseteq C$ & $C(x) \leftarrow P(x, y)$ & $P^{\mathcal{I}}(a, b) \leq C^{\mathcal{I}}(a)$ \\
\hline$\exists P^{-} . \top \sqsubseteq C$ & $C(y) \leftarrow P(x, y)$ & $P^{\mathcal{I}}(b, a) \leq C^{\mathcal{I}}(a)$ \\
\hline$P^{+} \sqsubset \bar{P}$ & $P(x, z) \leftarrow P(x, y) \wedge P(y, z)$ & $t\left(P^{\mathcal{I}}(a, b), P^{\mathcal{I}}(b, c)\right)<P^{\mathcal{I}}(a, c)$ \\
\hline$C \equiv D$ & $D(x) \leftarrow C(x)$ and $C(x) \leftarrow D(x)$ & $C^{\mathcal{I}}(a)=D^{\mathcal{I}}(a)$ \\
\hline$P \equiv Q$ & $P(x, y) \leftarrow Q(x, y)$ and $Q(x, y) \leftarrow P(x, y)$ & $P^{\mathcal{I}}(a, b)=Q^{\mathcal{I}}(a, b)$ \\
\hline$P \equiv Q^{-}$ & $P(x, y) \leftarrow Q(y, x)$ and $Q(y, x) \leftarrow P(x, y)$ & $P^{\mathcal{I}}(a, b)=Q^{\mathcal{I}}(b, a)$ \\
\hline$C_{1} \sqcap C_{2} \sqsubseteq D$ & $D(x) \leftarrow C_{1}(x) \wedge C_{2}$ & $t\left(C_{1}^{\mathcal{I}}(a), C_{2}^{\mathcal{I}}(a)\right) \leq D^{\mathcal{I}}(a)$ \\
\hline$C \sqsubseteq D_{1} \sqcap D_{2}$ & $D_{1}(x) \leftarrow C(x)$ and $D_{2}(x) \leftarrow C(x)$ & $C^{\mathcal{I}}(a) \leq D_{1}^{\mathcal{I}}(a)$ and $C^{\mathcal{I}}(a) \leq D_{2}^{\mathcal{I}}(a)(t=\min )$ \\
\hline$C_{1} \sqcup C_{2} \sqsubseteq D$ & $D(x) \leftarrow C_{1}(x)$ and $D(x) \leftarrow C_{2}(x)$ & $D^{\mathcal{I}}(a) \leq C_{1}^{\mathcal{I}}(a)$ and $D^{\mathcal{I}}(a) \leq C_{2}^{\mathcal{I}}(a)(u=\max )$ \\
\hline$D \sqsubseteq \forall P . C$ & $C(y) \leftarrow D(x) \wedge P(x, y)$ & $t\left(D^{\mathcal{I}}(b), P^{\mathcal{I}}(a, b)\right) \leq C^{\mathcal{I}}(a)(R$-implications $)$ \\
\hline$\exists P . C \sqsubseteq D$ & $D(x) \leftarrow P(x, y) \wedge C(x)$ & $t\left(P^{\mathcal{I}}(a, b), C^{\mathcal{I}}(b)\right) \leq D^{\mathcal{I}}(a)$ \\
\hline
\end{tabular}

further simplified to $t\left(P^{\mathcal{I}}(a, b), D^{\mathcal{I}}(a)\right) \leq C^{\mathcal{I}}(b)$. Since this fuzzy interpretation holds for every model we can educe the fuzzy def-Horn rule $C(y) \leftarrow P(x, y) \wedge D(x)$. Using the $S$ implication there can be no further simplification of $D^{\mathcal{I}}(a) \leq$ $\mathcal{J}\left(P^{\mathcal{I}}(a, b), C^{\mathcal{I}}(b)\right)$ and thus there can result no def-Horn rule. Consequently, we have shown that for the fuzzy $R$ implication there exists a semantic equivalence between the fuzzy Description Logics axiom $D \sqsubseteq \forall P$.C and the defHorn rule $C(y) \leftarrow P(x, y) \wedge D(x)$. In the same way we can show that the range restriction $\top \sqsubseteq \forall P$.C is equivalent to the def-Horn rule $C(y) \leftarrow P(x, y)$ only when the $R$-implication is used. However in order to express the range restriction we have previously used the axiom $\exists P^{-} . \top \sqsubseteq C$ that can be mapped to def-Horn rules without any restrictions.

When the universal quantifier occurs in the left hand side of a subclass axiom of the form $\forall P . C \sqsubseteq D$ its interpretation is $D^{\mathcal{I}}(a) \geq \inf _{b \in \Delta^{\mathcal{I}}} \mathcal{J}\left(P^{\mathcal{I}}(a, b), C^{\mathcal{I}}(b)\right)$. This expression gives us no information and moreover, the corresponding rule is not a fuzzy def-Horn rule so, we conclude that when the universal quantifier occurs in the left hand side of a subclass axiom there exists no mapping between f-OWL and fuzzy def-Horn rules.

4) Existential Restriction ( $\exists)$ : In Description Logics the existential quantifier (like the universal quantifier) can only be used in restrictions of the form $\exists P$.C. This is equivalent to a First Order Logic clause of the form $\exists y \cdot P(x, y) \wedge$ $C(y)$, which means that there exists a variable $y$ for which $P(x, y)$ is valid and that belongs to the concept $C$. An existential quantifier that occurs in the left hand side of a subclass axiom of the form $\exists P . C \sqsubseteq D$ is interpreted as $\sup _{b \in \Delta^{\mathcal{I}}} t\left(P^{\mathcal{I}}(a, b), C^{\mathcal{I}}(b)\right) \leq D^{\mathcal{I}}(a)$. This is equivalent to $t\left(P^{\mathcal{I}}(a, b), C^{\mathcal{I}}(b)\right) \leq D^{\overline{\mathcal{I}}}(a)$, as for every expression $x \geq \sup y$ and $x \geq y$ are equivalent. Thus, there exists semantic equivalence between the fuzzy axiom $\exists P . C \sqsubseteq D$ and the fuzzy def-Horn rule $D(x) \leftarrow P(x, y) \wedge C(x)$.

When the existential quantifier occurs in the right hand side of a subclass axiom of the form $D \sqsubseteq \exists P . C$ its interpretation is $\sup _{b \in \Delta^{\mathcal{I}}} t\left(P^{\mathcal{I}}(a, b), C^{\mathcal{I}}(b)\right) \geq D^{\mathcal{I}}(a)$. This interpretation gives us no information and therefore we cannot educe any def-Horn rule. This means that when the existential quantifier occurs in the left hand side of a subclass axiom there exists no mapping between f-OWL and fuzzy def-Horn rules.

5) Negation and Cardinality Restrictions $(\neg$, $\leqslant$ and $\geqslant)$ : These constructors cannot, in general, be mapped into fuzzy def-Horn. In case of negation this is obvious as fuzzy def-Horn rules do not allow negation in either the Head nor the Body of the rule. Moreover, cardinality restrictions correspond to assertions of variable equality and inequality in First Order Logic that is not supported by fuzzy def-Horn rules.

All the mappings that have been presented above are summarized in Table II, where $P, Q$ are roles, $C, C_{1}, \ldots, C_{n}, D$ and $D_{1}, \ldots, D_{n}$ are concept descriptors, $o$ and $o_{1}, \ldots, o_{n}$ are names of individuals, $a, b \in \Delta^{\mathcal{I}}$ are objects and $t$ is a t-norm for fuzzy intersection (conjunction) and $u$ is a t-conorm for fuzzy union (disjunction).

\section{DEFINING F-DLP}

From the above we can conclude that the Fuzzy Description Logics axioms $C \sqsubseteq D, A \equiv B, \exists P . \top \sqsubseteq C$, $\exists P^{-} \cdot \top \sqsubseteq C, P \sqsubseteq Q, P \equiv Q, P \equiv Q^{-}, P^{+} \sqsubseteq P$, $D \sqsubseteq \forall \bar{P} \cdot C, \exists P . \bar{C} \sqsubseteq D, o: C \geq \bar{r}, o: C \leq \bar{r}$, $\left\langle o_{1}, o_{2}\right\rangle: P \geq \bar{r}$ and $\left\langle o_{1}, o_{2}\right\rangle: P \leq \bar{r}$ can, in general, be mapped to fuzzy def-Horn rules without losing their semantics. However, the fact that the axiom $C \sqsubseteq D_{1} \sqcap D_{2}$ can be mapped without loss of its semantics only when the Gödel's t-norm (min) is used, the axiom $C_{1} \sqcup C_{2} \sqsubseteq D$ can be 
TABLE III

FUZZY DESCRIPTION LOGIC LANGUAGES AND FUZZY DESCRIPTION LOGICS AXIOMS

\begin{tabular}{l|c|c|c}
\hline \hline & $C \sqsubseteq D_{1} \sqcap D_{2}$ & $C_{1} \sqcup C_{2} \sqsubseteq D$ & $D \sqsubseteq \forall P . C$ \\
\hline $\mathrm{f}_{R-D H L}$ & & $\sqrt{ }$ \\
$\mathrm{f}_{R \min }-D H L$ & $\sqrt{ }$ & $\sqrt{ }$ & $\sqrt{ }$ \\
$\mathrm{f}_{R \max }-D H L$ & & $\sqrt{ }$ & $\sqrt{ }$ \\
$\mathrm{f}_{R \operatorname{minmax}}-D H L$ & $\sqrt{ }$ & & \\
$\mathrm{f}_{S}-D H L$ & & $\sqrt{ }$ & \\
$\mathrm{f}_{S \min }-D H L$ & $\sqrt{ }$ & $\sqrt{ }$ & \\
$\mathrm{f}_{S \max }-D H L$ & & $\sqrt{ }$ & \\
$\mathrm{f}_{\text {Sminmax }}-D H L$ & $\sqrt{ }$ &
\end{tabular}

mapped without loss of its semantics only when the Gödel's t-conorm $(\max )$ is used and that the axiom $D \sqsubseteq \forall P . C$ can be mapped without loss of its semantics only when we use $R$-implication, results to the definition of eight different languages, fuzzy $\mathrm{f}_{R}-D H L, \mathrm{f}_{R \min }-D H L, \mathrm{f}_{R \max }-D H L$, $\mathrm{f}_{R \operatorname{minmax}}-D H L, \mathrm{f}_{S}-D H L, \mathrm{f}_{S \min }-D H L, \mathrm{f}_{S \max }-D H L$, $\mathrm{f}_{\text {Sminmax }}-D H L$. These languages are defined according to what implication ( $R$ or $S$ ), what t-norm (min or other) and t-conorm (max or other) is used.

In Table III we present the fuzzy axioms that cannot be mapped without loss of their semantics for all the DLP languages. For each of the above languages we define a Description Logic Program based on this language, via the definition of a Description Horn Logic ontology, as follows.

An $f-D H L$ ontology is the set of fuzzy Description Horn Logic axioms that can be mapped to fuzzy def-Horn rules without loss of their semantics.

The mapping between fuzzy Description Logics and fuzzy def-Horn rules preserves the semantic equivalence. Let $\mathcal{K}$ be a fuzzy $f-D H L$ ontology and $\mathcal{H}$ be the fuzzy defHorn ruleset that results from applying the mapping to all the axioms of $\mathcal{K}$. Then the $\mathcal{H}$ set is logically equivalent to $\mathcal{K}$ with respect to the semantics of First Order Logic, which means that $\mathcal{H}$ has the same models as $\mathcal{K}$.

A fuzzy Description Logic Program $f-D L P$ is the fuzzy Logic Programming ruleset that are the logic equivalent of a fuzzy $f-D H L$ set.

\section{CONCLUSion}

In this paper we studied the mapping between fuzzy Description Logics and fuzzy def-Horn rules, based on the work done by Grosof, Horrocks, Volz and Decker [6] on mapping crisp Description Logic to def-Horn rules. Our contribution concerns the investigation of the mapping between fuzzy Description Logics and fuzzy LPs, and thus the definition of the various fragments of fuzzy Description Logic Programs. This is extremely important as the representation of vague and imprecise information in the Semantic Web gains more and more attention by the research community. Hence, following the current trend of research we have to find ways to combine fuzzy DLs with fuzzy LP systems in order to provide a complete framework for representing fuzziness in the Semantic Web. As far as we know there exists no such investigation of the fuzzy DLP fragment of fuzzy Description Logics.

\section{ACKNOWLEDGEMENT.}

This work was partially supported by the European Commission under project X-Media (FP6-26978). The work of Giorgos Stoilos was partially supported by the Greek Secretariat of Research and Technology (PENED Ontomedia 03 ED 475).

\section{REFERENCES}

[1] T. Berners-Lee, J. Hendler, and O. Lassila, "The semantic web," Scientific American, 2001.

[2] I. Horrocks, P. F. Patel-Schneider, and F. van Harmelen, "From $\mathcal{S H \mathcal { I }}$ and RDF to OWL: The making of a web ontology language," Web Semantics, vol. 1, no. 1, pp. 7-26, 2003.

[3] W3C, OWL Web Ontology Language, http://www.w3.org/2004/OWL/, 2003.

[4] I. Horrocks and P. Patel-Schneider, "Reducing OWL entailment to description logic satisfiability," in In Proc. of the 2nd International Semantic Web Conference(ISWC)., 2003.

[5] W3C, RDF Primer, http://www.w3.org/TR/rdf-primer/.

[6] B. N. Grosof, I. Horrocks, R. Volz, and S. Decker, "Description logic programs: Combining logic programs with description logic," in $W W W$ '03: Proceedings of the 12th international conference on World Wide Web, 2003.

[7] T. Eiter, T. Lukasiewicz, R. Schindlauer, and H. Tompits, "Combining answer set programming with description logics for the semantic web," in In Proc. of the International Conference of Knowledge Representation and Reasoning (KR'04), 2004.

[8] G. Stamou, V. Tzouvaras, J. Pan, and I. Horrocks, "A fuzzy extension of swrl," in W3C Workshop on Rule Languages for Interoperability, 2005, Position Paper.

[9] C. Matheus, "Using ontology-based rules for situation awareness and information fusion," in W3C Work. on Rule Languages for Interoperability, 2005, Position Paper.

[10] M. Kifer, "Requirements for an expressive rule language on the semantic web," in W3C Workshop on Rule Languages for Interoperability, 2005, Position Paper.

[11] U. Straccia, "Fuzzy description logic programs," in Proceedings of the 11th International Conference on Information Processing and Management of Uncertainty in Knowledge-Based Systems, (IPMU-06), 2006.

[12] F. Bobillo, M. Delgado, and J. Go'mez-Romero, "A crisp representation for fuzzy SHOIN with fuzzy nominals and general concept inclusions," in Proc. of the 2nd International Workshop on Uncertainty Reasoning for the Semantic Web (URSW 06), Athens, Georgia, 2006.

[13] L. A. Zadeh, "Fuzzy sets," Information and Control, vol. 8, pp. 338353, 1965.

[14] U. Straccia, "Towards a fuzzy description logic for the semantic web," in Proceedings of the 2nd European Semantic Web Conference, 2005.

[15] G. Stoilos, G. Stamou, and J. Pan, "Handling imprecise knowledge with fuzzy description logics," in Proceedings of the International Workshop on Description Logics (DL 2006), Lake District, UK, 2006.

[16] G. Stoilos, G. Stamou, V. Tzouvaras, J. Pan, and I. Horrocks, "Fuzzy OWL: Uncertainty and the semantic web," in Proc. of the International Workshop on OWL: Experiences and Directions, 2005. 